\title{
Histo- and Dynamorphisms Revisited
}

\author{
Ralf Hinze Nicolas $\mathrm{Wu}^{*}$ \\ Department of Computer Science \\ University of Oxford \\ $\{$ ralf.hinze,nicolas.wu\}@cs.ox.ac.uk
}

\begin{abstract}
Dynamic programming algorithms embody a widely used programming technique that optimizes recursively defined equations that have repeating subproblems. The standard solution uses arrays to share common results between successive steps, and while effective, this fails to exploit the structural properties present in these problems. Histomorphisms and dynamorphisms have been introduced to expresses such algorithms in terms of structured recursion schemes that leverage this structure. In this paper, we revisit and relate these schemes and show how they can be expressed in terms of recursion schemes from comonads, as well as from recursive coalgebras. Our constructions rely on properties of bialgebras and dicoalgebras, and we are careful to consider optimizations and efficiency concerns. Throughout the paper we illustrate these techniques through several worked-out examples discussed in a tutorial style, and show how a recursive specification can be expressed both as an array-based algorithm as well as one that uses recursion schemes.
\end{abstract}

Keywords dynamic programming, recursion schemes, histomorphisms, dynamorphisms

\section{Introduction}

Many important algorithms can be expressed using recursion equations where solutions are built up from recursive steps. In divideand-conquer algorithms such recursion equations can often be executed efficiently, since a problem is divided up into independent subproblems that are then solved recursively before being combined to form a final solution. However, in the particular case where a subproblem is repeated at different stages in the computation, recursion equations should be considered no more than a specification: the solution to each repeated subproblem is naively recomputed, often leading to exponential complexity that can be avoided. The key observation made by dynamic programming algorithms is that that solutions to subproblems can be memoised and reused when identical subproblems are later encountered, thus preventing the expense of needless recomputation.

The standard approach to implementing a dynamic programming algorithm is to use a table of values to store intermediate re-

\footnotetext{
* This work has been funded by EPSRC grant number EP/J010995/1.
}

[Copyright notice will appear here once 'preprint' option is removed.] sults. Such tables are usually indexed by the input parameters of the recursion, and are populated with values the first time a particular subproblem has been encountered, which are then reused when this subproblem is encountered again. In a functional programming language with lazy evaluation, such tables are easy to construct, since values are populated as they are demanded, which leads to implementations that closely resemble their recursive counterparts, but do not suffer from their inefficiencies.

Our goal is to show how categorically-inspired recursion schemes can be used to efficiently solve such problems, and we hope that this study will become useful to programmers who are interested in understanding how category theory can be used in the design of algorithms where the structure of the computation is important. This is not the first time that dynamic programming algorithms have been investigated from a categorical perspective. Histomorphisms have long been understood as a means of capturing courseof-value recursion, which is sufficient for some dynamic programming algorithms, where the recursion follows the pattern of the input data [13]. In particular, histomorphisms are constrained to work on problems where the input can be expressed as an initial algebra. However, not all problems follow such a rigid pattern, and dynamorphisms are a more general recursion scheme that was introduced to lift this restriction in the setting of CPO [9]. We extend this work by using recursive coalgebras to prove uniqueness within the setting of a wider range of categories.

In order to make this material accessible, we present a number of solutions to classic dynamic programming problems, and work through various solutions in a tutorial style. Our development is in Haskell [12], not only because the ensuing programs can be efficiently executed, but also because the language allows us to express solutions that closely resemble the categorical notions that underpin the theory.

On a more theoretical note, we also show how histomorphisms relate not only to one another, but also how they can be expressed as recursion schemes from comonads, and as recursion schemes from recursive coalgebras. Much of this work builds on the notions presented in [7], where distributive laws and bialgebras witness the correspondence between adjoint folds and recursion schemes from comonads. In this paper, we present an extensive case study based on that material, and show how further optimizations can be derived.

This paper makes the following novel contributions.

- We demonstrate how histomorphisms and dynamorphism can be applied to a number of different problems.

- We show how bialgebras relate histomorphisms to recursion schemes from comonads, and how dicoalgebras relate dynamorphisms to recursion schemes from recursive coalgebras.

- We use these relationships to derive optimized versions of histoand dynamorphisms. 
- We use type families to witness efficient implementations of inductive types from base functors in Haskell.

The work in this paper draws significantly from categorical machinery. As such, we assume that the reader has at least some basic knowledge of the categorical trinity: categories, functors and natural transformations. Aside from these, we also assume that the reader has an understanding of initial algebras and comonads; these notions will be introduced formally, but we will not linger long on these constructions. Aside from these, no further knowledge will be required, and we will introduce such notions as cofree comonads, distributive laws, bialgebras, and the (co)-EilenbergMoore category when they are required.

The paper is structured as follows. Section 2 introduces a number of dynamic programming algorithms that will be revisited throughout the paper. A brief overview of some of the basic concepts we use is given in Section 3. We introduce histomorphisms in Section 4 and relate these to recursion schemes from comonads in Section 5. We then introduce dynamorphisms in Section 6 and relate these to recursion schemes from recursive coalgebras in Section 7. Finally, we present related work in Section 8, and conclude in Section 9.

\section{Dynamic Programming}

Before looking at the construction of histo- and dynamorphisms we first take a look at at the different kinds of algorithms that fit under the umbrella of dynamic programming. Dynamic programming relies on the principle of optimality, where the optimal solution to a problem can be determined by first breaking the problem into subproblems, optimally solving those subproblems, and combining the ensuing subsolutions into a final answer.

The knapsack problem A classic example of of a dynamic algorithm is the unbounded knapsack problem. Suppose we are interested in maximizing the total value of elements that are placed into a knapsack with a fixed weight capacity. The elements are chosen from a set of items that are assigned a particular weight and value, each item being unbounded in number. We might represent the set of items as a list of pairs $(w, v)$, where $w$ is the weight and $v$ is the value. For instance, consider the following list:

$$
\begin{aligned}
& w v s::[(\mathbb{N}, \text { Double })] \\
& w v s=[(12,4),(1,2),(2,2),(1,1),(4,10)] .
\end{aligned}
$$

With a knapsack of capacity 15 the optimal solution is to choose three elements from the 2 nd and 5th items, for a total value of 36 .

This problem can be solved using a recursive function, that forms the basis of a specification:

$$
\begin{aligned}
& \text { knapsack }_{1}:: \mathbb{N} \rightarrow \text { Double }^{\text {knapsack }_{1} c=\text { maximum }_{0}} \\
& \quad\left[v+\text { knapsack }_{1}(c-w) \mid(w, v) \leftarrow w v s, 0<w \wedge w \leqslant c\right] .
\end{aligned}
$$

The value of a knapsack with capacity $c$ is determined by finding the item in wvs that maximizes the value of the knapsack when it has been added: an element of weight $w$ and value $v$ increases the value of a knapsack by $v$, and decreases its capacity by $w$. Only items with positive weight that can fit into the knapsack are considered. The function maximum $_{0}$ returns 0 when given an empty list, and otherwise returns the maximum value in the list.

This specification makes no attempt to be efficient, and naively recomputes the values of knapsacks with capacities that have already been explored. In order to avoid these recomputations, the intermediate results can be stored in a table that is populated by the recursion itself, and used to lookup values that have already been visited.

$$
\begin{aligned}
& \text { knapsack }_{2}:: \mathbb{N} \rightarrow \text { Double } \\
& \text { knapsack }_{2} n=\text { table } ! n \text { where } \\
& \quad \text { table }=\text { tabulate }(0, n) \text { knapsack } \\
& \quad \text { knapsack } c=\text { maximum }_{0} \\
& \quad[v+\text { table } !(c-w) \mid(w, v) \leftarrow w v s, 0<w \wedge w \leqslant c]
\end{aligned}
$$

This definition closely mirrors the specification, where the main

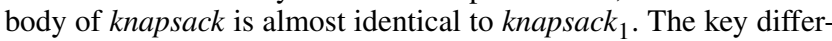
ence is that the recursive calls have been replaced by looking up values in a table. This table is constructed by the function tabulate, which takes as its arguments the bounds of the array that is to be constructed, as well as a function that produces values for given indices.

$$
\begin{aligned}
& \text { tabulate }::(I x i) \Rightarrow(i, i) \rightarrow(i \rightarrow a) \rightarrow \text { Array } i \text { a } \\
& \text { tabulate ixs } f=\text { array ixs }[(i, f i) \mid i \leftarrow \text { range ixs }]
\end{aligned}
$$

Thus the function knapsack and the array table are mutually recursive: values are initially tabulated by using the function knapsack, and knapsack makes use of the table to find values that have already been computed. This relies on lazy evaluation, where values are generated only as they are demanded: it is the calling convention that determines which values are calculated next. We shall see how this sets the pattern of how dynamic programming algorithms can be solved using arrays in the problems that follow. Note that there is an unfulfilled proof obligation here: one must show that the recursion is well-founded; it is precisely this proof that histomorphisms and dynamorphisms provide, and, as we shall see, we will have to work hard to transmogrify the original formulation into the form required by these schemes.

Catalan numbers A simple example of a course-of-value program that makes use of all its subcomponents is the evaluation of the Catalan numbers. Amongst other things, the Catalan numbers can be used to find the number of distinct well-formed arrangements that can be made with a set of $n$ matching parentheses. The recursive definition is as follows:

$$
\begin{aligned}
& \text { catalan }_{1}:: \mathbb{N} \rightarrow \mathbb{N} \\
& \text { catalan }_{1} 0=1 \\
& \text { catalan }_{1}(n+1)=\operatorname{sum}\left[\text { catalan }_{1} i * \text { catalan }_{1}(n-i) \mid i \leftarrow[0 \ldots n]\right]
\end{aligned}
$$

For example, the value of catalan $_{1} 3$ is 5 , which can be seen through a simple enumeration of the possibilities:

$$
\text { () () (), () (()), (()) (), (() ()), ((())) . }
$$

The recursive solution works by considering all of the different ways of splitting an expression with parentheses.

Strictly speaking this is not a dynamic programming problem, since we are not seeking an optimal solution. However, it does exhibit the same hallmarks: common subproblems are encountered time and again, and there is scope to share solutions between recursive calls to increase the efficiency of this algorithm. As before, we apply the technique dynamic programming, where array-based memoisation is used to store and share results.

$$
\begin{aligned}
& \text { catalan }_{2}:: \mathbb{N} \rightarrow \mathbb{N} \\
& \text { catalan }_{2} p=\text { table } ! p \text { where } \\
& \quad \text { table }=\text { tabulate }(0, p) \text { catalan } \\
& \quad \text { catalan } 0=1 \\
& \quad \text { catalan }(n+1)=\text { sum }[\text { table } ! i * \text { table } !(n-i) \mid i \leftarrow[0 \ldots n]]
\end{aligned}
$$

Again this implementation builds an array that is indexed in place of recursive calls.

Chain matrix multiplication The chain matrix multiplication problem concerns finding the minimal number of operations required to multiply a chain of matrices of arbitrary length. The multiplication of a $p \times q$ matrix by a $q \times r$ matrix yields a matrix of 
size $p \times r$ in $p q r$ scalar operations. This multiplication is associative, yielding the same result regardless of the order in which more than two matrices are multiplied. However it is easy to show that different parenthesisations can lead to different costs. For example, consider multiplying a chain of three matrices of sizes $2 \times 3,3 \times 5$, and $5 \times 7$. There are two solutions, where multiplying the first two matrices and then the third costs 100 operations, whereas multiplying the last two matrices and then the first costs 147 operations.

The naive solution to this problem is to compute the cost of all possible parenthesisations. This algorithm takes time proportional to the Catalan numbers to generate all the different sequences, each of which is checked in isolation. We can improve upon this solution by using dynamic programming, where the results for any (sub)-parenthesisation is calculated only once and reused where appropriate. As usual, we start with a recurrence equation that solves the problem. We assume that the matrices $A_{1} \ldots A_{n}$ are given to be multiplied, and matrix $A_{k}$ has dimensions given by $a_{k-1} \times a_{k}$.

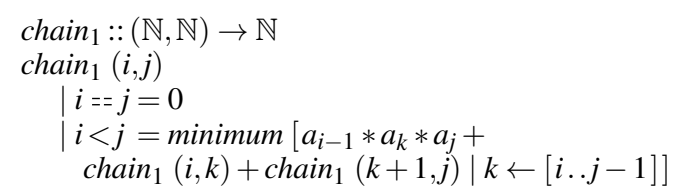

(In a sense, the specification is already geared towards an imperative array-based solution: the argument to the recursion is represented as a pair, which is an efficient representation of a contiguous segment when the data is globally stored in an array.) This solution makes use of the principle of optimality, by noting that the optimal solutions to subproblems can be combined to form the final solution. In this case, the optimal chain for multiplying matrices $A_{i} \ldots A_{j}$ is given by finding the value $k$ that minimizes the number of scalar operations, when the optimal values for chaining matrices $A_{i} \ldots A_{k}$ and $A_{k+1} \ldots A_{j}$ are known. The final answer for this is held in $\operatorname{chain}_{1}(1, n)$, where $n$ is the number of matrices that are being multiplied.

To turn this into a more efficient array-based version, we employ the usual technique and memoise the results of the recursion:

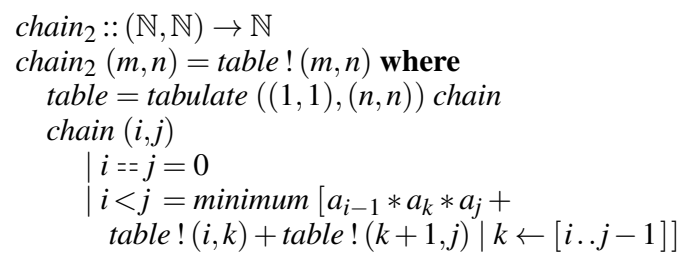

Although the recursive definition is in two variables, we can capture this quite simply by creating a multi-dimensional array. Note that all the complexity is hidden in the function tabulate, which is overloaded on the type of indices.

The bitonic travelling-salesman problem The bitonic travellingsalesman problem is a variation of the classic NP-hard travellingsalesman problem: given a set of points and distances between each pair of points, the task is to find the shortest route that visits each point exactly once before returning back to the start. The variation is that the points are assumed to be on a plane, and share no $x$ coordinate, and furthermore, the solutions are restricted to consider only bitonic tours: paths that start at the leftmost point, then move strictly towards the rightmost point, and then strictly left again back to the start, having covered all points. For convenience we assume that there are $m$ points, $x_{0} \ldots x_{m}$, ordered by their $x$ coordinate. We denote the distance between the points $x_{i}$ and $x_{j}$ by $\overline{x_{i} x_{j}}$. Under these circumstances, we can show that the optimal path can be found in $O\left(\mathrm{~m}^{2}\right)$ time.

First we present a solution that uses strong induction on the naturals. We ensure that the value of bitonic $_{1} n$ is the length of the shortest tour that includes all the points $x_{0} \ldots x_{n+1}$. The base case is bitonic $_{1} 0$, which is simply twice the distance between the first two points. For the inductive case, we assume that all values bitonic $_{1} i$ contain the shortest path when $i \leqslant n$, and show how to find the shortest path for bitonic $1(n+1)$. If we consider the point $n+2$, at the far right of the tour, then this must be connected to the point $n+1$, and also to some other point $k \leqslant n$. Since the tour is bitonic, this implies that the path also connects the points $k+1 \ldots n$ in succession. Therefore, a bitonic tour with $n+1$ at its rightmost is given by bitonic $k$ plus these connections, and minus the path between $k$ and $k+1$. We are obviously interested in finding the shortest tour given by some $k$. This is expressed precisely in the following recursively defined function:

$$
\begin{aligned}
& \text { bitonic }_{1}:: \mathbb{N} \rightarrow \text { Double } \\
& \text { bitonic }_{1} 0 \quad=2 * \overline{x_{0} x_{1}} \\
& \text { bitonic }_{1}(n+1)=\text { minimum }\left[\text { bitonic }_{1} k-\overline{x_{k} x_{k+1}}+\overline{x_{k} x_{n+2}}\right. \\
& \left.\quad+\operatorname{sum}\left[\overline{x_{i} x_{i+1}} \mid i \leftarrow[k+1 \ldots n+1]\right] \mid k \leftarrow[0 \ldots n]\right]
\end{aligned}
$$

Using this recurrence, the solution is found in bitonic $_{1}(m-1)$. To turn this into an efficient version we must memoise the results of bitonic $_{1} k$, to avoid the recomputation of subsolutions. We omit this definition, since it is similar to the array-based solutions of the previous examples.

A second solution to the bitonic travelling-salesman problem can be formulated that has quite a different invariant. The recursion equation for bitonic $_{2} i j$ expresses the minimal traversal of the points that starts at $x_{i}$, travels strictly left to $x_{0}$, and then strictly right to $x_{j}$. We assume that $i \leqslant j$, and that all points smaller than $j$ are in the path. Clearly, when $i==j$ we have a cycle: the final answer is to be found in bitonic $m \mathrm{~m}$.

$$
\begin{aligned}
& \text { bitonic }_{2}::(\mathbb{N}, \mathbb{N}) \rightarrow \text { Double } \\
& \text { bitonic }_{2}(0,0)=0 \\
& \text { bitonic }_{2}(0,1)=\overline{x_{0} x_{1}} \\
& \text { bitonic }_{2}(i, j) \\
& \qquad \mid \begin{array}{l}
i<j-1=\text { bitonic }_{2}(i, j-1)+\overline{x_{j-1} x_{j}} \\
\quad \mid \text { otherwise }^{\prime}=\text { minimum }\left[\text { bitonic }_{2}(k, i)+\overline{x_{k} x_{j}} \mid k \leftarrow[0 \ldots i-1]\right]
\end{array}
\end{aligned}
$$

There are two base cases for this recursion. The first is the tour that contains only the point $x_{0}$, which has a distance of 0 . The second base case is a path that connects $x_{0}$ and $x_{1}$, which has length $\overline{x_{0} x_{1}}$. Now we consider a path from $x_{i}$ to $x_{j}$. When $i<j-1$, then we must connect $x_{j-1}$ and $x_{j}$, since our invariant is that all points less than $j$ are in the path, so this distance is added to the result of bitonic $_{2} i(j-1)$. Otherwise, either $i==j-1$ or $i==j$, and in both cases we find the minimal path that has one end at $i$, and the other end going through some $k$ and immediately to $j$. To turn this into an efficient version we must construct a table in two dimensions.

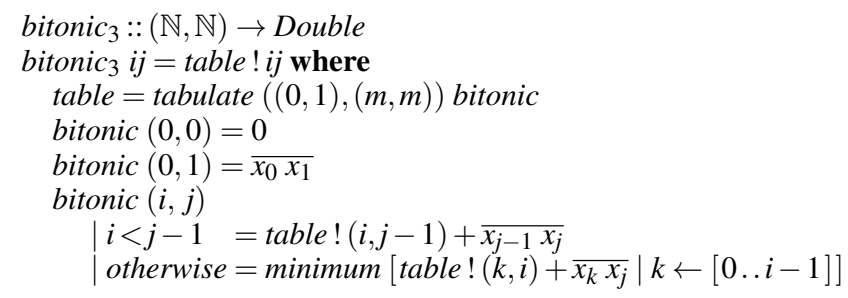

Again this efficient version follows quite naturally from the recursive specification.

In each of these examples, we turn a recursive definition into a more efficient array-based solution that solves the problem. However, this is unsatisfactory in the sense that we have no guarantee that the recursion is well-defined. In the remainder of the paper we will focus on recursion schemes where the structure of the lookup table comes from the data itself. 


\section{Background}

In this section we introduce some of the basic concepts that will be used in the remainder of the paper, and show how these notions can be implemented in Haskell.

Algebras and coalgebras Algebras and coalgebras form the basis for the categorical description of many recursion schemes. Given an endofunctor $\mathrm{F}: \mathscr{C} \rightarrow \mathscr{C}$, an $\mathrm{F}$-algebra is a pair $(a, A)$, where $a: \mathrm{F} A \rightarrow A$ is an arrow and $A: \mathscr{C}$ is an object, which are known as the action and carrier of the algebra. (This deviates a little from the standard notation $(A, a)$, since it gives us syntax that distinguishes algebras from coalgebras.) An F-homomorphism between algebras $(a, A)$ and $(b, B)$ is an arrow $h: A \rightarrow B: \mathscr{C}$ such that $h \cdot a=b \cdot \mathrm{F} h$.

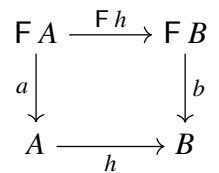

F-homomorphisms compose and have an identity, so it follows that F-algebras and F-homomorphisms form a category, which we call $\mathrm{F}-\mathrm{Alg}(\mathscr{C})$. The initial object of this category, if it exists, is given by (in, $\mu \mathrm{F})$ and called the initial $\mathrm{F}$-algebra. The initiality implies that to each F-algebra, $(a, A)$, there exists a unique F-homomorphism, $(a):($ in,$\mu \mathrm{F}) \rightarrow(a, A)$, called a fold. The algebra in is, in fact, an isomorphism, so $\mu \mathrm{F}$ is a fixed-point of $\mathrm{F}$ (the least fixed-point), a fact known as Lambek's lemma [11].

Dually, given an endofunctor $\mathrm{G}: \mathscr{C} \rightarrow \mathscr{C}$, a G-coalgebra is a pair $(C, c)$, where $C: \mathscr{C}$ is the carrier and $c: C \rightarrow \mathrm{G} C$ is the action of the coalgebra. A G-homomorphism between coalgebras $(C, c)$ and $(D, d)$ is an arrow $h: C \rightarrow D: \mathscr{C}$ that satisfies $\mathrm{G} h$. $c=d \cdot h$. Just as before, a category G-Coalg $(\mathscr{C})$ can be formed from G-coalgebras and G-homomorphisms. The final object of this category, if it exists, is given by $(\nu \mathrm{G}, o u t)$ and called the final $\mathrm{G}$-coalgebra. The unique homomorphism to the $\mathrm{G}$-algebra $(C, c)$, called an unfold, is written $\llbracket c]: C \rightarrow \vee \mathrm{G}$.

The category F-Alg $(\mathscr{C})$ has more structure than $\mathscr{C}$. The forgetful or underlying functor $\mathrm{U}^{\mathrm{F}}: \mathrm{F}-\mathrm{Alg}(\mathscr{C}) \rightarrow \mathscr{C}$ forgets about the additional structure: $\mathrm{U}^{\mathrm{F}}(a, A)=A$ and $\mathrm{U}^{\mathrm{F}} h=h$. An analogous functor can be defined for coalgebras: $\mathrm{U}_{\mathrm{G}}: \mathrm{G}-\operatorname{Coalg}(\mathscr{C}) \rightarrow \mathscr{C}$.

Inductive types Since the action of an initial algebra is an isomorphism, initial algebras can be used to assign a semantics to recursively defined datatypes. As an example, the inductive datatype

$$
\text { data Tree }=\text { Empty } \mid \text { Node Tree } \mathbb{N} \text { Tree }
$$

is modelled by the initial algebra (in, $\mu$ Tree), where the so-called base functor

\section{data Tree tree $=$ Empty $\mid$ Node tree $\mathbb{N}$ tree}

abstracts away from the recursive occurrences of Tree. Note that we use a different font to distinguish the base functor from the inductive datatype. The Haskell rendering of the isomorphism in, the action of the initial algebra,

$$
\begin{aligned}
& \text { in :: Tree Tree } \rightarrow \text { Tree } \\
& \text { in (Empty) }=\text { Empty } \\
& \text { in (Node lar) }=\text { Nodel ar }
\end{aligned}
$$

amounts to a simple renaming of constructors.

Turning to the semantics of recursively defined functions, a simple tree consumer such as

$$
\begin{aligned}
& \text { depth }:: \text { Tree } \rightarrow \mathbb{N} \\
& \text { depth }(\text { Empty })=0 \\
& \text { depth }(\text { Node la } r)=1+(\text { depth l'max'depth } r)
\end{aligned}
$$

can be modelled by the fold (depth), where the algebra

$$
\begin{aligned}
& \text { depth :: Tree } \mathbb{N} \rightarrow \mathbb{N} \\
& \text { depth (Empty) }=0 \\
& \text { depth (Node } d l a d r)=1+(d l \text { ' } \max \text { ' } d r)
\end{aligned}
$$

is a non-recursive function that maps the results of the recursive calls to the overall result.

The standard approach to implementing this machinery in Haskell is to make in a data constructor of a generic fixed-point constructor: data $\mu \mathrm{F}=\operatorname{In}\left\{i^{\circ}:: \mathrm{F}(\mu \mathrm{F})\right\}$. We depart from this approach, and instead use Haskell's type classes and family synonyms [3] to witness this isomorphism. Thus, for inductive types, we introduce the following class:

$$
\begin{aligned}
& \text { class }(\text { Functor } \mathrm{F}) \Rightarrow \text { Inductive } \mathrm{F} \text { where } \\
& \text { type } \mu \mathrm{F}:: * \\
& \text { in }:: \mathrm{F}(\mu \mathrm{F}) \rightarrow \mu \mathrm{F} \\
& \text { in }:: \mu \mathrm{F} \rightarrow \mathrm{F}(\mu \mathrm{F}) \\
& (-)::(\mathrm{F} a \rightarrow a) \rightarrow(\mu \mathrm{F} \rightarrow a) .
\end{aligned}
$$

Here, in is implemented as a function rather than a data constructor, and there is an obligation on the implementor to ensure that in and $i n^{\circ}$ are indeed inverses. However, it is possible to define $i n^{\circ}$ in terms of a fold, and vice versa, so these form suitable default implementations:

$$
\begin{aligned}
& i n^{\circ}=(\text { fmap in }\rangle \\
& (a)=a \cdot f m a p(a) \cdot i n^{\circ}
\end{aligned}
$$

This allows us to keep the implementation of the fixed point abstract, and in turn, gives us the freedom to associate efficient representations to base functors.

This is particularly useful for primitive types such as natural numbers. The base functor for these is expressed by Nat:

$$
\begin{aligned}
& \text { data Nat } n=\text { Zero } \mid \text { Succ } n \\
& \text { instance Functor Nat where } \\
& \quad \text { fmap } f \text { Zero }=\text { Zero } \\
& \quad \text { fmap } f(\text { Succ } x)=\text { Succ }(f x) .
\end{aligned}
$$

The Inductive instance for this datatype is simply the natural numbers, which are implemented efficiently as integers in Haskell:

$$
\begin{aligned}
& \text { type } \mathbb{N}=\text { Int } \\
& \text { instance Inductive Nat where } \\
& \text { type } \mu \mathrm{Nat}=\mathbb{N} \\
& \text { in Zero }=0 \\
& \text { in }(\text { Succ } n)=n+1 \\
& \text { in }^{\circ} 0=\text { Zero } \\
& i^{\circ}(n+1)=\text { Succ } n \text {. }
\end{aligned}
$$

This lets us freely use properties of the structure of natural numbers without being too heavily penalized.

Comonads Functional programmers have embraced monads, and to a lesser extent, comonads, to capture effectful and contextsensitive computations. We shall use comonads to model 'recursive calls in context'. A comonad is a functor $\mathrm{N}: \mathscr{C} \rightarrow \mathscr{C}$ equipped with a natural transformation $\epsilon: \mathrm{N} \rightarrow \mathrm{Id}$ (counit), that extracts a value from a context, and a second natural transformation $\delta: N \rightarrow \mathrm{N} \circ \mathrm{N}$ (comultiplication) that duplicates a context. These functions are subject to the comonad laws:

$$
\begin{aligned}
& (\epsilon \circ N) \cdot \delta=N, \\
& (N \circ \epsilon) \cdot \delta=N, \\
& (\delta \circ N) \cdot \delta=(N \circ \delta) \cdot \delta .
\end{aligned}
$$

Here we use categorical notation, where natural transformations can be composed horizontally ( $($ ), and vertically $(\cdot)$, and the identity natural transformation for a functor is denoted by the functor itself. 
The first two properties, the counit laws, state that duplicating a context and then discarding a duplicate is the same as doing nothing. The third property, the coassociative law, equates the two ways of duplicating a context twice.

\section{Histomorphisms}

Dynamic programming algorithms make use of solutions to previously visited subproblems to compute the values of new ones. In other words, values that are computed are placed in some context, and then extracted from that context when needed. As a first approximation, this pattern is captured by a histomorphism, which has access to the whole history of a computation. Recall that a fold is made available the result of the recursive calls on the immediate substructures. By contrast, a histomorphism can resort to the results of the recursive calls on all substructures.

Before we go into the details of how a histomorphism is defined, we first introduce the so-called cofree comonad of a functor $F$, which we write as $F_{\infty}$. This comonad serves to provide the context in which results are placed during recursive calls. Loosely speaking, it serves as a generic counterpart of the memo tables implemented by arrays above.

Cofree comonad Categorically speaking, the cofree comonad comes from the following adjunction between the category of coalgebras and its underlying category:

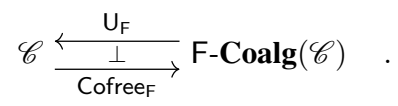

The forgetful functor $\mathrm{U}_{\mathrm{F}}$ has a right adjoint Cofree $_{\mathrm{F}}$ that maps an object $A$ to the cofree coalgebra Cofree $A=\left(\mathrm{F}_{\infty} A\right.$, tail $\left._{\infty} A\right)$. Very generally speaking, Cofree $F_{F}$ can be used to capture the behaviour of 'systems'. It may help to think of the functor $F$ as a static description of all possible transitions for a class of different systems, and of the object $A$ as a type of system states. The elements of $\mathrm{F}_{\infty} A$ then capture the entire behaviour of a system as the infinite unfolding of all possible transitions. The action of the cofree coalgebra tail $_{\infty}: \mathrm{F}_{\infty} A \rightarrow \mathrm{F}\left(\mathrm{F}_{\infty} A\right)$ maps such a description to the $\mathrm{F}$-structure of all possible successor systems.

The adjunction provides further infrastructure: the counit $\epsilon$ which we denote head ${ }_{\infty} \mathrm{F}_{\infty} A \rightarrow A$ extracts the initial state of a system; given a state-transition function expressed as an $\mathrm{F}$-coalgebra $a: A \rightarrow \mathrm{F} A$, the unit $\eta$ which we write $\eta(A, a)=\left\lfloor a \rrbracket: A \rightarrow \mathrm{F}_{\infty} A\right.$ constructs the infinite unfolding from a given initial state. This data satisfies an important property, which establishes a bijection between certain arrows in $\mathscr{C}$ and certain arrows in F-Coalg $(\mathscr{C})$. Specifically, an F-coalgebra homomorphism $g:(A, a) \rightarrow$ Cofree $_{\mathrm{F}} B$ is uniquely determined by a mapping $f: A \rightarrow B$ from states of type $A$ to observations of type $B$. This so-called universal property can be neatly expressed as an equivalence:

$$
f=\operatorname{head}_{\infty} B \cdot g \Longleftrightarrow \mathrm{F}_{\infty} f \cdot \llbracket a \mathbf{\rrbracket}=g
$$

for all arrows $f: A \rightarrow B$ and homomorphisms $g:(A, a) \rightarrow$ Cofree $_{\mathrm{F}} B$.

Now, every adjunction induces a comonad [8]. The adjunction (2) gives rise to the cofree comonad $F_{\infty}=U_{F} \circ$ Cofree $_{F}$.

The cofree comonad in Haskell One can show that final coalgebras and cofree coalgebras are interdefinable. In one direction we have $v \mathrm{~F} \cong \mathrm{F}_{\infty} 1$ where 1 is the final object. In the other direction we have $\mathrm{F}_{\infty} A \cong v X . A \times \mathrm{F} X$, which forms the basis for an implementation in Haskell. Using Haskell's higher-kinded datatypes, $\mathrm{F}_{\infty} A$ can be readily implemented as follows.

$$
\begin{aligned}
& \text { data } \mathrm{F}_{\infty} a=\text { Cons }_{\infty}\left\{\text { head }_{\infty}:: \text { a, } \text { tail }_{\infty}:: \mathrm{F}\left(\mathrm{F}_{\infty} a\right)\right\} \\
& \text { instance }(\text { Functor } \mathrm{F}) \Rightarrow \text { Functor }\left(\mathrm{F}_{\infty}\right) \text { where } \\
& \quad \text { fmap } f\left(\text { Cons }_{\infty} \text { a ts }\right)=\text { Cons }_{\infty}(\text { f } a)(\text { fmap }(\text { fmap } f) \text { ts })
\end{aligned}
$$

Here, Cons $_{\infty}$ is the inverse of the isomorphism head ${ }_{\infty} \Delta$ tail $_{\infty}$. The type $F_{\infty}$ can be seen as the type of generalized streams of observations-it behaves as a 'stream' because each successive layer has a head $_{\infty}$ that contains a value, and 'generalized' because the 'tail' is an F-structure of 'streams' rather than just a single one. A generalized stream is, in fact, very similar to a generalized rose tree, except that the latter is usually seen as an element of an inductive type, whereas this construction is patently coinductive. If we instantiate the base functor of the cofree comonad to Id, we obtain the type of simple streams.

Given a coalgebra $a: A \rightarrow \mathrm{F} A$, the implementation of the unit 【a】: $a \rightarrow \mathrm{F}_{\infty} a$ is fairly straightforward, where the function cons $_{\infty}::$ $\left(a, \mathrm{~F}\left(\mathrm{~F}_{\infty} a\right)\right) \rightarrow \mathrm{F}_{\infty} a$ is the uncurried version of Cons $_{\infty}$ :

$$
\begin{aligned}
& \left\lfloor-】::(\text { Functor } \mathrm{F}) \Rightarrow(a \rightarrow \mathrm{F} a) \rightarrow\left(a \rightarrow \mathrm{F}_{\infty} a\right)\right. \\
& \llbracket a \rrbracket=h \text { where } h=\text { cons }_{\infty} \cdot(\text { id } \triangle \text { fmap } h \cdot a) .
\end{aligned}
$$

This takes an initial seed that is used to create the head of the structure, and is also combined with the algebra to recursively grow the next level of values in the tails.

As its name suggests, the cofree comonad is comonadic, and so comes equipped with a means of extracting a value from its context, $\epsilon=$ head $_{\infty}$, and a means of duplicating a context, $\delta=$ 【tail $)_{\infty} \rrbracket$, which uses an entire $F_{\infty}$-structure as the state.

We have noted above that $\mathrm{Id}_{\infty}$ yields the type of streams. A more interesting base functor is Nat which gives rise to the type Nat of $_{\infty}$ non-empty colists. For example, the call【 $i n^{\circ} 】 2$ generates the colist

$$
\text { Cons } \left._{\infty} 2\left(\text { Succ }\left(\text { Cons }_{\infty} 1 \text { (Succ }\left(\text { Cons }_{\infty} 0 \text { Zero }\right)\right)\right)\right) \text {. }
$$

This corresponds to the list of all predecessors of 2 .

Histomorphisms With these basics in place, we are now ready to give the original formulation of histomorphisms [13].

The argument to a histomorphism is a 'context-sensitive' algebra $a: \mathrm{F}\left(\mathrm{F}_{\infty} A\right) \rightarrow A$ that works on a structure that contains all the recursive subsolutions, and combines these to form a new solution of type $A$. Informally, $\mathrm{F}_{\infty} A$ is a hierarchical memo-table where each successive level contains a subsolution of type $A$ together with an F-substructure; the deepest level is the base case of the datatype.

A histomorphism is defined to be the unique solution $x: \mu \mathrm{F} \rightarrow A$ of the equation:

$$
x \cdot \text { in }=a \cdot \mathrm{F}\left(\mathrm{F}_{\infty} x \cdot\left\lfloor i n^{\circ}\right)\right) .
$$

The coalgebra $\left(i n^{\circ} 】: \mu \mathrm{F} \rightarrow \mathrm{F}_{\infty}(\mu \mathrm{F})\right.$ turns an element of an inductive type into a table of all substructures. The histomorphism is recursively applied to each of the substructures, making the results of the recursive calls readily available to the algebra $a$.

Remark. There are a number of different variations of this definition, depending on how the argument of $F$ is expressed. In the definition above we have used a formulation that is based on the unit of the adjunction (2): $h=\mathrm{F}_{\infty} x \cdot\left\lfloor i n^{\circ}\right.$ 】. The original characterization in [13] is recovered if we identify $\mathrm{F}_{\infty} A$ and $\vee X . A \times \mathrm{F} X$, thus giving us $h=\llbracket x \Delta i n^{\circ} \rrbracket$.

Equation (4) specifies the notion of an histomorphism, however, it does not serve as a blue-print for an efficient implementation. Indeed, as an implementation it is exponential in the sense that the annotated tree is recomputed at every recursive call. Also, at the outset it is not clear that the equation (4) has a unique solution. We postpone both issues until Section 5 where we attack them in a more general setting.

Histomorphisms in Haskell We can turn the naive definition of a histomorphism into a recursion scheme in Haskell by making the most of the fact that in has an inverse, $i{ }^{\circ}$, which is provided by the Inductive typeclass:

$$
\begin{aligned}
& \text { histo }_{1}::(\text { Inductive } \mathrm{F}) \Rightarrow\left(\mathrm{F}\left(\mathrm{F}_{\infty} a\right) \rightarrow a\right) \rightarrow(\mu \mathrm{F} \rightarrow a) \\
& \text { histo }_{1} a=x \text { where } x=a \cdot \text { fmap }\left(\text { fmap } x \cdot\left\lfloor\text { in }^{\circ} \rrbracket\right) \cdot \text { in }^{\circ} .\right.
\end{aligned}
$$


The histomorphism first deconstructs a recursive type to expose one level of its base functor. The function $\left(i n^{\circ}\right\rfloor:: \mu \mathrm{F} \rightarrow \mathrm{F}_{\infty}(\mu \mathrm{F})$ is then applied to the functor arguments, turning them into a table of predecessors, before the histomorphism is recursively applied with fmap (histo 1 a) to produce subsolutions as labels to each level. Finally, the algebra $a$ takes this structure that contains all subsolutions, and combines them to form a final solution.

\section{Recursion Schemes From Comonads}

Histomorphisms involve both an algebra and a coalgebra, and combine them in an interesting way. We have noted above that $\left\langle i n^{\circ}\right\rangle$ is a coalgebra, but it is actually a bit more: it is a coalgebra for the comonad $\mathrm{F}_{\infty}$. Furthermore, the algebra in and the coalgebra $\left(i n^{\circ} 】\right.$ go hand-in-hand. They are related by a so-called distributive law $\lambda$ : $\mathrm{F} \circ \mathrm{F}_{\infty} \rightarrow \mathrm{F}_{\infty} \circ \mathrm{F}$ and form what is known as a $\lambda$-bialgebra, a combination of an algebra and a coalgebra with a common carrier. In particular, in and $i n^{\circ} 】$ satisfy the so-called pentagonal law.

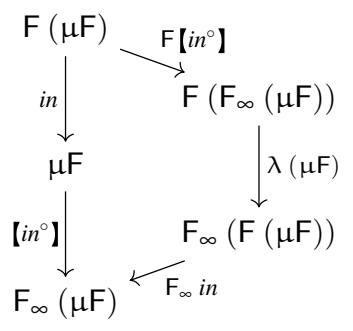

Loosely speaking, the distributive law $\lambda: \mathrm{F} \circ \mathrm{F}_{\infty} \rightarrow \mathrm{F}_{\infty} \circ \mathrm{F}$ defined

$$
\lambda A=\mathrm{F}_{\infty}\left(\mathrm{F}\left(\operatorname{head}_{\infty} A\right)\right) \cdot\left[\mathrm{F}\left(\operatorname{tail}_{\infty} A\right)\right]
$$

allows us to swap the functors $F$ and $F_{\infty}$.

The cofree comonad is by no means special. In fact, histomorphisms are an instance of recursion schemes from comonads [14]. We postpone a formal introduction of the scheme after we have provided the necessary background in the following section, which can be skipped by those already familiar with the material.

\subsection{Background}

Coalgebras for a comonad A coalgebra for a comonad $\mathrm{N}$ is an $\mathrm{N}$-coalgebra $(C, c)$ that respects $€$ and $\delta$ :

$$
\begin{aligned}
& \epsilon C \cdot c=i d_{C}, \\
& \delta C \cdot c=\mathrm{N} c \cdot c .
\end{aligned}
$$

If we first create a context using $c$ and then focus, we obtain the original value. Creating a nested context is the same as first creating a context and then duplicating it. For example, the so-called cofree coalgebra $(\mathrm{N} C, \delta C)$ is respectful, which follows directly from (1b) and (1c).

Coalgebras that respect $\epsilon$ and $\delta$ and $\mathrm{N}$-coalgebra homomorphisms form a category, known as the (co)-Eilenberg-Moore category and denoted $\mathscr{C}_{\mathrm{N}}$.

Eilenberg-Moore construction As noted above, every adjunction generates a comonad. The converse is also true: every comonad $\mathrm{N}$ induces an adjunction that generates $\mathrm{N}$-in fact, in two canonical ways. One construction was discovered by Kleisli [10], the other by Eilenberg and Moore [4]. We shall need the latter, which constructs a right adjoint to the forgetful functor $U_{N}: \mathscr{C}_{N} \rightarrow \mathscr{C}$.

$$
\mathscr{C} \underset{\frac{U_{N}}{\text { Cofree }_{N}}}{\stackrel{\perp}{L}} \mathscr{C}_{N}
$$

The functor Cofree $\mathrm{N}$ maps an object to the cofree coalgebra for $\mathrm{N}$ :

$$
\begin{aligned}
\text { Cofree }_{\mathrm{N}} B & =(\mathrm{N} B, \delta B), \\
\text { Cofree }_{\mathrm{N}} f & =\mathrm{N} f .
\end{aligned}
$$

The adjunction establishes a bijection between certain arrows in $\mathscr{C}$ and certain arrows in $\mathscr{C}_{\mathrm{N}}$. Specifically, an N-coalgebra homomorphism $h:(A, a) \rightarrow$ Cofree $_{\mathrm{N}} B$ is uniquely determined by an arrow $f: A \rightarrow B$ in $\mathscr{C}$. As before, this universal property can be expressed as an equivalence:

$$
f=\epsilon B \cdot h \quad \Longleftrightarrow \mathrm{N} f \cdot a=h,
$$

for all arrows $f: A \rightarrow B$ and homomorphisms $h:(A, a) \rightarrow(\mathrm{N} B, \delta B)$. The homomorphism $h$ is also called the transpose of $f$ and is denoted $\lfloor f\rfloor=\mathrm{N} f \cdot a$. Conversely, $f$ is the transpose of $h$, denoted $\lceil h\rceil=\epsilon B \cdot h$.

Eilenberg-Moore categories generalize categories of coalgebras: we have $\mathrm{F}$-Coalg $(\mathscr{C}) \cong \mathscr{C}_{N}$ where $\mathrm{N}=\mathrm{F}_{\infty}$ is the cofree comonad. In particular, F-coalgebra homomorphisms are in 1-1 correspondence to $\mathrm{N}$-coalgebra homomorphisms:

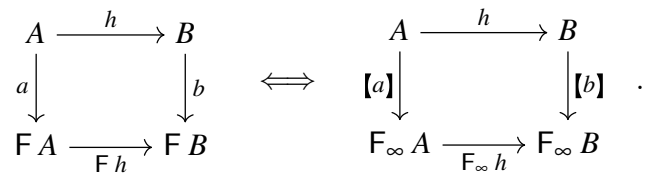

Note that the isomorphism F-Coalg $(\mathscr{C}) \cong \mathscr{C}_{\mathrm{N}}$ implies that $[a 】$ is always a coalgebra for the comonad $F_{\infty}$. Conversely, each respectful $\mathrm{F}_{\infty}$-coalgebra is of this form.

Distributive laws A distributive law $\lambda: \mathrm{F} \circ \mathrm{N} \rightarrow \mathrm{N} \circ \mathrm{F}$ of an endofunctor $\mathrm{F}$ over a comonad $\mathrm{N}$ is a natural transformation satisfying the two coherence conditions:

$$
\begin{aligned}
& (\epsilon \circ F) \cdot \lambda=F \circ \epsilon, \\
& (\delta \circ F) \cdot \lambda=(N \circ \lambda) \cdot(\lambda \circ N) \cdot(F \circ \delta) .
\end{aligned}
$$

The first law has type $\mathrm{F} \circ \mathrm{N} \rightarrow \mathrm{F}$, and states that there are two equivalent ways of extracting a value from a comonadic context that is nested in a functor: either by first exposing the comonad to the outside by applying a distributive law, and then extracting the functorial value from the comonadic context; or by working directly inside the functor, and extracting a value from the comonadic context that is there. The second law has type $\mathrm{F} \circ \mathrm{N} \rightarrow \mathrm{N} \circ \mathrm{N} \circ \mathrm{F}$ and states that pushing a functorial value into a context and then duplicating the context is equivalent to first duplicating the context embedded in a functor, and then shifting the functorial inside the contexts.

One can show that the distributive law $\lambda: \mathrm{F} \circ \mathrm{F}_{\infty} \rightarrow \mathrm{F}_{\infty} \circ \mathrm{F}$ defined in Equation (6) obeys these laws; the proof is beyond the scope of this paper.

Bialgebras A bialgebra combines an algebra and a coalgebra with a common carrier. Bialgebras come in many flavours; we need the variant that combines F-algebras and coalgebras for a comonad N. The two functors have to interact coherently, described by a distributive law.

Let $\lambda: \mathrm{F} \circ \mathrm{N} \rightarrow \mathrm{N} \circ \mathrm{F}$ be a distributive law for the endofunctor $\mathrm{F}$ over the comonad N. A $\lambda$-bialgebra $(a, X, c)$ consists of an $\mathrm{F}$ algebra $a$ and a coalgebra $c$ for the comonad $\mathrm{N}$ such that the pentagonal law holds:

$$
c \cdot a=\mathrm{N} a \cdot \lambda X \cdot \mathrm{F} c .
$$

Loosely speaking, this law allows us to swap the algebra $a$ and the coalgebra $c$. A $\lambda$-bialgebra homomorphism is both simultaneously an F-algebra and an N-coalgebra homomorphism. 
The pentagonal law (12) has two asymmetric renderings, which identify the algebra $a$ and the coalgebra $c$ as homomorphisms.

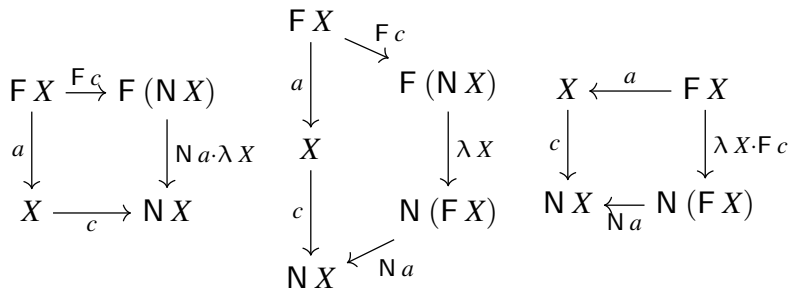

The diagram on the left shows that $c$ is an F-algebra homomorphism. Dually, the diagram on the right identifies $a$ as an $\mathrm{N}$ coalgebra homomorphism.

\subsection{Recursion Schemes from Comonads}

Now that the terminology is in place, we are in a position to generalize histomorphisms to recursion schemes from comonads [14]. These form a general recursion principle that makes use of a comonad $\mathrm{N}$ to provide 'contextual information' to the algebra of the to-be-defined function.

Let $\lambda: \mathrm{F} \circ \mathrm{N} \rightarrow \mathrm{N} \circ \mathrm{F}$ be a distributive law, and let $($ in, $\mu \mathrm{F}, c)$ be a $\lambda$-bialgebra. For any $(\mathrm{F} \circ \mathrm{N})$-algebra $(b, B)$ there is a unique arrow $f: \mu \mathrm{F} \rightarrow B$ such that

$$
f \cdot \text { in }=b \cdot \mathrm{F}(\mathrm{N} f \cdot c) .
$$

The composition $\mathrm{N} f \cdot c$ creates a context that makes the results of recursive calls available to the algebra $b$. Note that $b$ is a 'contextsensitive' algebra - an $(\mathrm{F} \circ \mathrm{N})$-algebra, rather than merely an $\mathrm{F}$ algebra.

One way to prove uniqueness is to use the Eilenberg-Moore adjunction (9) to relate solutions of (13) to certain $\lambda$-bialgebra homomorphisms. Abstracting away from in and identifying $\mathrm{N} f \cdot c$ as the transpose of $f$, one can establish the following equivalence

$$
f \cdot a=b \cdot \mathrm{F} h \quad \Longleftrightarrow \quad h \cdot a=\lfloor b\rfloor \cdot \mathrm{F} h
$$

where $h=\lfloor f\rfloor=\mathrm{N} f \cdot c$ is the transpose of $f$. The diagrammatical rendering makes explicit that $h$ is not only an F-algebra homomorphism but also a $\lambda$-bialgebra homomorphism.
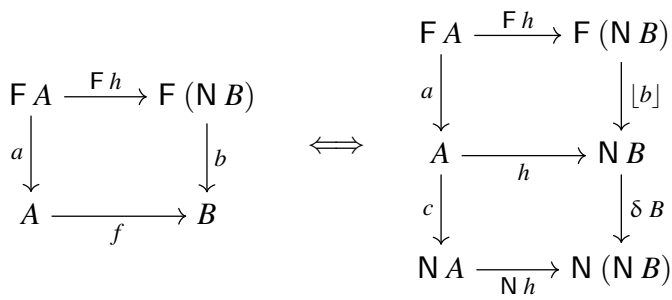

For the proof of this fact we refer to [7]. Now, if $a$ is in, the action of the initial algebra, the homomorphism $h$ is uniquely defined, and hence $f$, as well.

Histomorphisms revisited To show that the original formulation of histomorphisms is an instance of this scheme, we must show that in and $\left(i n^{\circ}\right.$ ) form a $\lambda$-bialgebra, where $\lambda: \mathrm{F} \circ \mathrm{F}_{\infty} \rightarrow \mathrm{F}_{\infty} \circ \mathrm{F}$. To this end we make use of the following 1-1 correspondence between idbialgebras and $\lambda$-bialgebras, which is a consequence of the fact that
$\mathrm{F}-\operatorname{Coalg}(\mathscr{C}) \cong \mathscr{C}_{\mathrm{N}}$

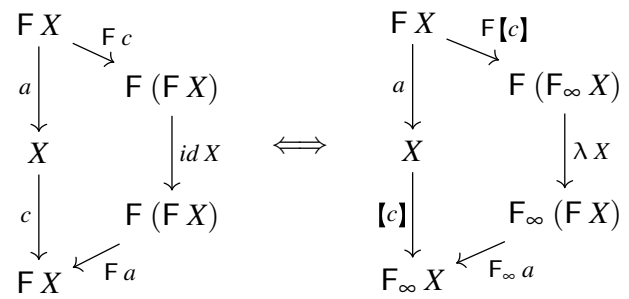

Note that for $i d$-bialgebras there is no coherence requirement on the $\mathrm{F}$-coalgebra as $\mathrm{F}$ is just a functor. Furthermore, recall that $\lfloor c\rceil$ is always a coalgebra for the comonad $F_{\infty}$ and that each respectful $F_{\infty}$-coalgebra is of this form.

The proof obligation that $\left(i n, \mu \mathrm{F},\left[i n^{\circ}\right]\right)$ is a $\lambda$-bialgebra is now easy to discharge.

$$
\begin{aligned}
& \left\lfloor i n^{\circ}\right\rfloor \cdot \text { in }=\mathrm{F}_{\infty} \text { in } \cdot \lambda(\mu \mathrm{F}) \cdot \mathrm{F}\left\lfloor i n^{\circ}\right\rfloor \\
& \Longleftrightarrow\{(16)\} \\
& i n^{\circ} \cdot \text { in }=\mathrm{F} \text { in } \cdot i d(\mu \mathrm{F}) \cdot \mathrm{F} \text { in }{ }^{\circ} \text {. }
\end{aligned}
$$

The latter equation holds trivially.

Efficiency improvements We have noted before that (4) is merely a specification of a histomorphism. Even though it is executable, it is not fit for public consumption as it implements the naive recursive definition, which often leads to an exponential running time. In a sense, the original definitions of knapsack and friends suffer from two problems: First, it is not clear that the recursion equations have a solution-framing the algorithm as an instance of (4) solves this problem; and second, as a program the recursion equations are horribly inefficient-this problem is what we tackle next.

Because of the 1-1 correspondence (14) we can implement $f: \mu \mathrm{F} \rightarrow B$ in terms of $h: \mu \mathrm{F} \rightarrow \mathrm{F}_{\infty} B$, which constructs an entire table of answers: $f=\lceil h\rceil=$ head $_{\infty} B \cdot h=$ head $_{\infty} B \cdot(\lfloor b\rfloor)$. So it remains to derive an efficient implementation of $\lfloor b\rfloor$. To this end observe that $\left(|b|, \mathrm{F}_{\infty} B, \delta B\right)$ forms a $\lambda$-bialgebra, which is also related to an $i d$-bialgebra.

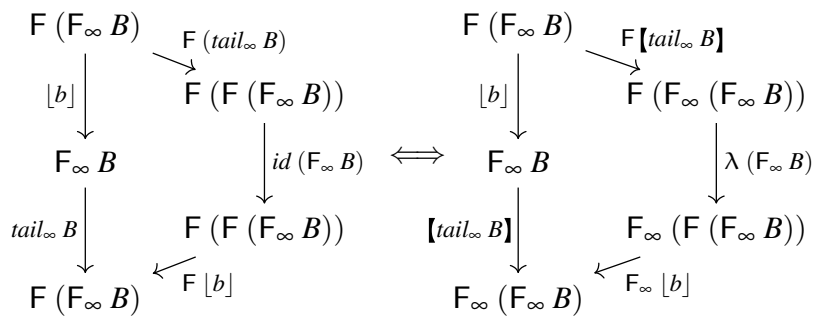

The diagram on the left identifies $\lfloor b\rfloor$ as an F-coalgebra homomorphism: tail $_{\infty} B \cdot\lfloor b\rfloor=\mathrm{F}\lfloor b\rfloor \cdot \mathrm{F}\left(\right.$ tail $\left._{\infty} B\right)$. Since furthermore head $_{\infty} B \cdot\lfloor b\rfloor=b$ using (9), we can invoke the universal property of cofree coalgebras (3) and conclude

$$
\lfloor b\rfloor=\mathrm{F}_{\infty} b \cdot\left\lfloor\mathrm{F}\left(\text { tail }_{\infty} B\right)\right\rfloor .
$$

Consequently, the histomorphism $f$ is given by

$$
f=\text { head }_{\infty} B \cdot\left(\mathrm{F}_{\infty} b \cdot\left\lfloor\mathrm{F}\left(\text { tail }_{\infty} B\right) \mathbf{I}\right) .\right.
$$

Loosely speaking, we have managed to turn the exponential specification into an implementation with a quadratic running-time. The fold makes a single sweep through the input structure; for each level the context-sensitive algebra $b$ is mapped over the table to create a table for the next level of recursion. (Of course, all of this depends on the particulars of $\mathrm{F}$ and $b$, which is why we said "loosely".) 
Ideally, we would like $b$ to be invoked only once per level. Interestingly, we can achieve this goal if we make use of the fact that that $h$ is a $\lambda$-bialgebra homomorphism, a blend of an $\mathrm{F}$ algebra and an $F_{\infty}$-coalgebra homomorphism. Furthermore recall that $F$-coalgebra homomorphisms are in 1-1 correspondence to $F_{\infty}$ coalgebra homomorphisms (10).
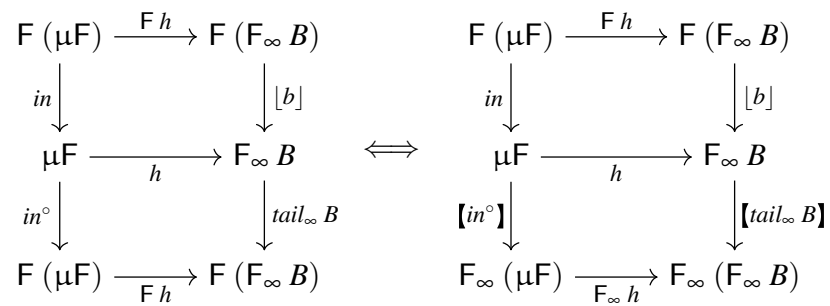

Now we use the fact that $h$ is both an F-algebra and an F-coalgebra homomorphism.

$$
\begin{aligned}
& h \cdot \text { in }=\lfloor b\rfloor \cdot \mathrm{F} h \wedge \mathrm{F} h \cdot \text { in }^{\circ}=\text { tail }_{\infty} B \cdot h \\
& \Longrightarrow\{\text { Leibniz }\} \\
& \operatorname{head}_{\infty} B \cdot h \cdot \text { in } \operatorname{head}_{\infty} B \cdot\lfloor b\rfloor \cdot \mathrm{F} h \wedge \operatorname{tail}_{\infty} B \cdot h=\mathrm{F} h \cdot \text { in }^{\circ} \\
& \Longleftrightarrow\left\{\operatorname{head}_{\infty} B \cdot\lfloor b\rfloor=b(9) \text { and in isomorphism }\right\} \\
& \operatorname{head}_{\infty} B \cdot h \cdot \text { in }=b \cdot \mathrm{F} h \wedge \operatorname{tail}_{\infty} B \cdot h \cdot \text { in }=\mathrm{F} h \\
& \Longleftrightarrow\{\text { products }\}
\end{aligned}
$$

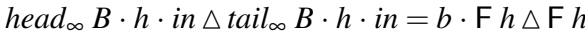

$$
\begin{aligned}
& \Longleftrightarrow \quad \text { fusion }\} \\
& \left(\text { head }_{\infty} B \triangle \operatorname{tail}_{\infty} B\right) \cdot h \cdot \text { in }=(b \triangle i d) \cdot \mathrm{F} h \\
& \Longleftrightarrow\left\{\text { head }_{\infty} B \triangle \text { tail }_{\infty} B \text { isomorphism }\right\} \\
& h \cdot \text { in }=\text { cons }_{\infty} \cdot(b \triangle i d) \cdot \mathrm{F} h
\end{aligned}
$$

Thus, $h=\left(\right.$ cons $\left._{\infty} \cdot(b \triangle i d)\right)$ and consequently

$$
f=\operatorname{head}_{\infty} B \cdot\left(\text { cons }_{\infty} \cdot(b \triangle i d) D .\right.
$$

Finally, we have arrived at an efficient formulation of histomorphisms, which is in fact equivalent to the definition of histomorphisms in [13], though a much shorter proof. This final solution folds over the input in a single sweep with $h$, which returns the entire history in a context. Once this is done, the head of this context is extracted. Note that this definition does not depend on lazyness.

Histomorphisms in Haskell revisited The implementation of more efficient histomorphisms in Haskell translates easily from the categorical notation.

$$
\begin{aligned}
& \text { histo }_{2}::(\text { Inductive } \mathrm{F}) \Rightarrow\left(\mathrm{F}\left(\mathrm{F}_{\infty} b\right) \rightarrow b\right) \rightarrow(\mu \mathrm{F} \rightarrow b) \\
& \text { histo }_{2} b=\text { head }_{\infty} \cdot\left(\text { cons }_{\infty} \cdot(b \triangle i d)\right)
\end{aligned}
$$

This works by ensuring that the input value has an inductive type, and folds this value in a single sweep into a structure of type $\mathrm{F}_{\infty} b$, where the head contains the final solution.

\subsection{Examples}

Let us now apply the framework of histomorphisms to the examples presented in Section 2. As we have seen, histomorphisms are defined to work on input types that are initial algebras. This is certainly the case for knapsack ${ }_{1}$ and catalan $_{1}$, since the initial algebra in question is simply the natural numbers. On the other hand, bitonic $_{1}$ and chain $_{1}$ cannot be expressed as histomorphisms, since the input to these functions, $(\mathbb{N}, \mathbb{N})$, is not an initial algebra.

As we shall see, there is one important modification that needs to be made when translating the specifications to this version of the algorithms: indices in both the specifications and the tabular versions are absolute in the sense that they are indexed from some fixed origin that is taken as a reference point. With histomorphisms, the reference point is the 'current' point of call in the recursion, and so indices that refer to subsolutions are relative.

Knapsack problem To express the knapsack problem as a histomorphism, we will need to consider the input parameter as an initial algebra. This is more easily seen when we specialize the recursive specification to expose the structure of natural numbers in $c$ :

$$
\begin{aligned}
& \text { knapsack }_{3}:: \mathbb{N} \rightarrow \text { Double } \\
& \text { knapsack }_{3} 0=0 \\
& \text { knapsack }_{3}(c+1)=\text { maximum }_{0} \\
& \quad\left[v+\text { knapsack }_{3}(c-w) \mid(w+1, v) \leftarrow w v s, w \leqslant c\right]
\end{aligned}
$$

Just as in the array-based version of knapsack ${ }_{2}$, we will create an algebra that replaces the recursive call in the body of the algorithm with a lookup. This time, however, we will be looking up values in the cofree structure of the naturals, $\mathrm{Nat}_{\infty} v$, rather than an array.

To turn this into a histomorphism, we provide a contextsensitive algebra knapsack that uses the results of previous subsolutions found in the $\mathrm{Nat}_{\infty} v$ structure and returns the solution. This solution is then used by histo 2 which embeds this value at the top of the context that is used in the next round of the recursion.

$$
\begin{aligned}
& \text { knapsack }_{4}:: \mathbb{N} \rightarrow \text { Double } \\
& \text { knapsack }_{4}=\text { histo }_{2} \text { knapsack where } \\
& \text { knapsack }: \text { Nat }\left(\text { Nat }_{\infty} \text { Double }\right) \rightarrow \text { Double } \\
& \text { knapsack (Zero) }=0 \\
& \text { knapsack }(\text { Succ table })=\text { maximum }_{0} \\
& {\left[v+u \mid(w+1, v) \leftarrow w v s, \text { Just } u \leftarrow\left[\text { lookup }_{\infty} \text { table } w\right]\right]}
\end{aligned}
$$

When knapsack is called for the first time, the lookup table is Zero, and contains no elements, and the result is simply 0 . For each successive call, knapsack has access to previous computed values in table, one for each smaller knapsack capacity than the one currently in consideration.

Note that we have adjusted the indices of the lookup: in the original version, the recursive call is performed with knapsack ( $c-$ $w$ ), which is the absolute position of the knapsack capacity minus the weight of a given item. In the histomorphism version, we replace the lookup with the value $u$, which is the result of a relative indexing, where lookup $_{\infty} t(c-(c-w))=$ lookup $_{\infty} t w$. This works out nicely because the 'current' capacity $c$ is not available. Another difference is that the out of bounds guard $w \leqslant c$ has been replaced by Just $u \leftarrow[\ldots]$. The maximum is then calculated just as in the recursive version.

In order to find values in the $\mathrm{Nat}_{\infty} v$ structure, we introduce function lookup $p_{\infty}$, which provides access to the results stored in the head:

$$
\begin{aligned}
& \text { lookup }_{\infty}:: \mathrm{Nat}_{\infty} a \rightarrow \mathbb{N} \rightarrow \text { Maybe } a \\
& \text { lookup }_{\infty}\left(\text { Cons }_{\infty} a_{-}\right) \quad 0 \quad=\text { Just } a \\
& \text { lookup }_{\infty}\left(\text { Cons }_{\infty} a(\text { Zero })\right) \quad(n+1)=\text { Nothing } \\
& \text { lookup } \left._{\infty}\left(\text { Cons }_{\infty} \text { a (Succ as }\right)\right)(n+1)=\text { lookup }_{\infty} \text { as } n
\end{aligned}
$$

The effect of lookup $p_{\infty}$ table $n$ is to return the result that was computed $n$ 'steps' before the current point of call, since more recent values are found at the head of a Cons $s_{\infty}$ constructor.

Catalan numbers The generation of Catalan numbers proves to be an instructive example, since it is not expressible as a histomorphism. First notice that there is a slight twist, where each successive value makes use of all of the subsolutions: this can be seen in the definition of catalan $_{2}$, where computing the value of catalan $_{2}(n+1)$ must access all of the values with indices in the range $[0 \ldots n]$. It may then be tempting to admit the following bogus definition as a histomorphism, where a Catalan number is simply the result of the convolution, that is, summing the multiplication of 
a list of prior elements with its reversal.

$$
\begin{aligned}
& \text { catalan }_{3}:: \mathbb{N} \rightarrow \mathbb{N} \quad \text {-- WRONG } \\
& \text { catalan }_{3}=\text { histo }_{2} \text { catalan where } \\
& \text { catalan :: Nat }\left(\operatorname{Nat}_{\infty} \mathbb{N}\right) \rightarrow \mathbb{N} \\
& \text { catalan Zero } \quad=1 \\
& \text { catalan }(\text { Succ table })=\operatorname{sum}(\text { zipWith }(*) x s(\text { reverse } x s)) \\
& \text { where } x s=\text { elems }_{\infty} \text { table }
\end{aligned}
$$

At each step of the histomorphism the table in scope contains only the solutions to subproblems, so instead of pulling out values from the table using an index, we can instead select all of the elements at once, returning a list of all the previously computed solutions. So, why is this function bnot well-defined?

The function at fault is $e l e m s_{\infty}$, that extracts all of the values:

$$
\begin{array}{ll}
\text { elems }_{\infty}:: \text { Nat }_{\infty} v \rightarrow[v] & -- \text { WRONG } \\
\text { elem }_{\infty}\left(\text { Con }_{\infty} a(\text { Zero })\right. & =[a] \\
\text { elem } \left._{\infty}\left(\text { Cons }_{\infty} \text { a (Succ as }\right)\right) & =a: \text { elems }_{\infty} \text { as }
\end{array}
$$

This definition is bogus because we are converting the coinductive $\mathrm{Nat}_{\infty} v$ to a simple inductive list. Of course, this does not work in general. We shall revisit the Catalan numbers later, and show how they can be expressed as a dynamorphism.

\section{Dynamorphisms}

Histomorphisms insist that the input is an element of some initial algebra. Looking back at Section 2 we note that this is the case for some but not all of the examples: chain $_{1}$ and bitonic $_{2}$, for instance, take a pair of natural numbers as input. For these examples dynamorphisms come to the rescue. The basic idea is simple, but far reaching:

When we implemented histomorphisms

$$
x \cdot \text { in }^{\circ}=a \cdot \mathrm{F}\left(\mathrm{F}_{\infty} x \cdot\left\lfloor\operatorname{in}^{\circ} \rrbracket\right),\right.
$$

we made most of the fact that in has an inverse, turning the algebra on the left into a coalgebra on the right:

$$
x=a \cdot \mathrm{F}\left(\mathrm{F}_{\infty} x \cdot \llbracket i n^{\circ} \rrbracket\right) \cdot i n^{\circ} .
$$

The idea of dynamorphisms is to replace $i n^{\circ}$ by a so-called recursive coalgebra $c$.

$$
x=a \cdot \mathrm{F}\left(\mathrm{F}_{\infty} x \cdot \mathbf{\lfloor} \mathbf{】}\right) \cdot c .
$$

Loosely speaking, recursiveness guarantees that the equation still has a unique solution. We shall say more about recursive coalgebras in Section 7.1.

Remark. Dynamorphisms were originally introduced in the setting of partial orders and continuous functions with no restriction on the coalgebra $c$ [9]. Under these assumptions (17) has only a canonical solution, not a unique solution. We do not wish to go down this route.

As with histomorphisms, it is useful to abstract away from the cofree comonad $F_{\infty}$ and develop the recursion scheme in a more general setting. Before we do so, we record an implementation of this (inefficient) version of dynamorphisms in Haskell.

Dynamorphisms in Haskell The implementation is

$$
\begin{aligned}
& \text { dyna }_{1}::(\text { Functor } \mathrm{F}) \Rightarrow\left(\mathrm{F}\left(\mathrm{F}_{\infty} a\right) \rightarrow a\right) \rightarrow(c \rightarrow \mathrm{F} c) \rightarrow(c \rightarrow a) \\
& \text { dyna }_{1} \text { a } c=x \text { where } x=a \cdot \text { fmap }(\text { fmap } x \cdot\lfloor c \rrbracket) \cdot c .
\end{aligned}
$$

As with the definition of histo ${ }_{1}$, this is not efficient, since the intermediary structure is built in exponential time.

\section{Recursion Schemes From Recursive Coalgebras}

Histomorphisms combine an algebra and a coalgebra. For dynamorphisms we have transmogrified the algebra into a coalgebra.
Thus, dynamorphisms combine two coalgebras, of which one is a coalgebra for a comonad. As before, the two ingredients are related by the distributive law $\lambda: \mathrm{F} \circ \mathrm{F}_{\infty} \dot{\rightarrow} \mathrm{F}_{\infty} \circ \mathrm{F}$.<smiles>FC(F)(F)C(F)(F)C(F)(F)C(F)(F)F</smiles>

This is essentially the same diagram as in (5), only that the arrows previously labelled with in and $\mathrm{F}_{\infty}$ in have been flipped. There is no established name for the resulting structure. Capretta et al. [2] have coined the combination of two coalgebras a $\lambda$-dicoalgebra. We adopt the terminology, even though this is likely to cause confusion (there are also dialgebras, which are entirely different beasts).

As usual, we postpone a formal introduction of the scheme after we have provided the necessary background.

\subsection{Background}

Hylomorphisms and recursive coalgebras A hylomorphism (or algebra-from-coalgebra homomorphism) is a recursion scheme that captures the essence of divide-and-conquer algorithms. Such algorithms have three phases: first, a problem is broken into subproblems by a coalgebra $c: C \rightarrow \mathrm{F} C$; second, sub-problems are recursively turned into sub-solutions; and finally, sub-solutions are combined by an algebra $a: \mathrm{F} A \rightarrow A$ to form a solution. An arrow $h: C \rightarrow A$ is a hylomorphism, $h:(C, c) \rightarrow(a, A)$, if it satisfies

$$
h=a \cdot \mathrm{F} h \cdot c .
$$

A coalgebra $(C, c)$ is recursive (or algebra-initial) if for every algebra $(a, A)$ there is a unique hylomorphism $(C, c) \rightarrow(a, A)$ satisfying (18). An important recursive coalgebra is $\left(\mu \mathrm{F}, i n^{\circ}\right)$, which is also the final recursive coalgebra. Thus, using recursive coalgebras allows us to generalize the development of histomorphisms, which are a special case of dynamorphisms where the coalgebra is $i n^{\circ}$.

Dicoalgebras Let $\lambda: \mathrm{F} \circ \mathrm{N} \rightarrow \mathrm{N} \circ \mathrm{F}$ be a distributive law for the endofunctor $\mathrm{F}$ over the comonad $\mathrm{N}$. A $\lambda$-dicoalgebra $(X, c, d)$ consists of an F-coalgebra $c$ and a coalgebra $d$ for the comonad $\mathrm{N}$ such that the pentagonal law holds:

$$
\mathrm{N} c \cdot d=\lambda X \cdot \mathrm{F} d \cdot c .
$$

The pentagonal law (19) also has an asymmetric rendering, which identifies the coalgebra $c$ as an $\mathrm{N}$-coalgebra homomorphisms.

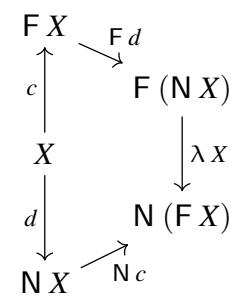

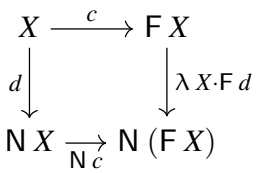

\subsection{Recursion Schemes From Recursive Coalgebras}

Let $\lambda: \mathrm{F} \circ \mathrm{N} \rightarrow \mathrm{N} \circ \mathrm{F}$ be a distributive law, and let $(C, c, d)$ be a $\lambda$-dicoalgebra where $c: C \rightarrow \mathrm{F} C$ is recursive. For any $(\mathrm{F} \circ \mathrm{N})$ algebra $(a, A)$ there is a unique arrow $f: C \rightarrow A$ such that

$$
f=a \cdot \mathrm{F}(\mathrm{N} f \cdot d) \cdot c .
$$


Quite amazingly, everything we said about histomorphisms and recursion schemes from comonads generalizes to this more expressive setting. In particular, there is a 1-1 correspondence between two kinds of hylomorphisms:

$$
f=a \cdot \mathrm{F} h \cdot c \Longleftrightarrow h=\lfloor a\rfloor \cdot \mathrm{F} h \cdot c
$$

where $h=\lfloor f\rfloor=\mathrm{N} f \cdot d$ is the transpose of $f$. Since the coalgebra $c$ is recursive, the equation on the right has a unique solution and hence the original equation (21), as shown by [2].

Dynamorphisms revisited To show that dynamorphisms are an instance of this scheme, we have to prove that $c$ and $\lfloor c 】$ form a $\lambda$ dicoalgebra, where $\lambda: F \circ F_{\infty} \rightarrow F_{\infty} \circ \mathrm{F}$. Like for bialgebras, there is a 1-1 correspondence between $i d$-dicoalgebras and $\lambda$-dicoalgebras.

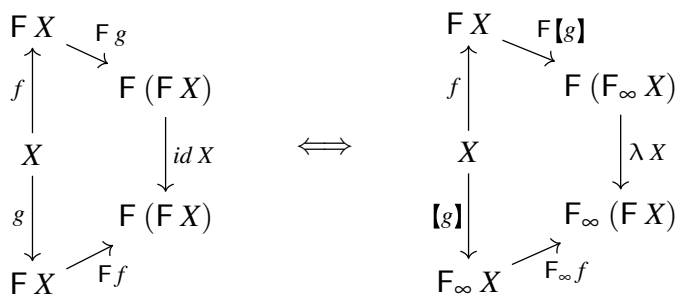

Using this property, the proof that $(C, c,\lfloor c\rfloor)$ forms a $\lambda$-dicoalgebra is a one-liner.

$$
\begin{aligned}
& \mathrm{F}_{\infty} c \cdot \llbracket c 】=\lambda X \cdot \mathrm{F}\lfloor c\rfloor \cdot c \\
& \{(22)\} \\
& \mathrm{F} c \cdot c=i d X \cdot \mathrm{F} c \cdot c
\end{aligned}
$$

Thus dynamorphisms are indeed an instance of the scheme above. In addition, histomorphisms are also an instance, since they are simply the case where we specialize further and instantiate $C:=\mu \mathrm{F}$ and $c:=i n^{\circ}$.

Efficiency improvements First of all, note that $\left(\lfloor a\rfloor, \mathrm{F}_{\infty} A, \delta A\right)$ still forms a $\lambda$-bialgebra and that $h$ can be seen as an arrow from a $\lambda$-dicoalgebra to a $\lambda$-bialgebra, see the diagram on the right below.
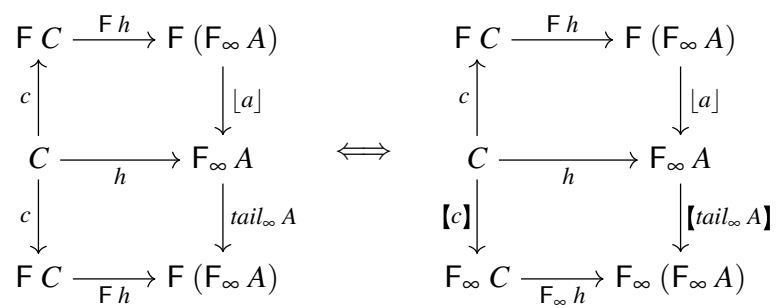

Again, we leverage on the fact that F-coalgebra homomorphisms are in 1-1 correspondence to $F_{\infty}$-coalgebra homomorphisms (10). The derivation of an efficient implementation of $h$ follows the template laid out in Section 5.2-the proof below is even somewhat simpler, even though it establishes a more general result.

$$
\begin{aligned}
& h=\lfloor a\rfloor \cdot \mathrm{F} h \cdot c \wedge \text { tail }_{\infty} \cdot h=\mathrm{F} h \cdot c \\
\Longrightarrow & \{\text { Leibniz } \\
& \text { head }_{\infty} \cdot h=\text { head }_{\infty} \cdot\lfloor a\rfloor \cdot \mathrm{F} h \cdot c \wedge \text { tail }_{\infty} \cdot h=\mathrm{F} h \cdot c \\
\Longleftrightarrow & \left\{\text { head }_{\infty} \cdot\lfloor a\rfloor=a(9)\right\} \\
& \text { head }_{\infty} \cdot h=a \cdot \mathrm{F} h \cdot c \wedge \text { tail }_{\infty} \cdot h=\mathrm{F} h \cdot c \\
\Longleftrightarrow & \{\text { products }\} \\
& \text { head } \cdot h \triangle \text { tail }_{\infty} \cdot h=a \cdot \mathrm{F} h \cdot c \triangle \mathrm{F} h \cdot c \\
\Longleftrightarrow & \{\text { fusion }\} \\
& \left(\text { head }_{\infty} \triangle \text { tail }_{\infty}\right) \cdot h=(a \triangle \text { id }) \cdot \mathrm{F} h \cdot c \\
\Longleftrightarrow & \left\{\text { head }_{\infty} \triangle \text { tail }_{\infty} \text { isomorphism }\right\} \\
& h=\text { cons }_{\infty} \cdot(\text { a } \triangle \text { id }) \cdot \mathrm{F} h \cdot c
\end{aligned}
$$

Since $c$ is a recursive coalgebra, the last equation has a unique solution.

Dynamorphisms in Haskell revisited The translation of this categorical machinery into Haskell is entirely straightforward:

$$
\begin{aligned}
& \text { dyna }_{2}::(\text { Functor } \mathrm{F}) \Rightarrow\left(\mathrm{F}\left(\mathrm{F}_{\infty} a\right) \rightarrow a\right) \rightarrow(c \rightarrow \mathrm{F} c) \rightarrow(c \rightarrow a) \\
& \text { dyna }_{2} \text { a } c=\text { head }_{\infty} \cdot h \text { where } h=\text { cons }_{\infty} \cdot(a \Delta i d) \cdot \text { fmap } h \cdot c .
\end{aligned}
$$

\subsection{Examples}

Dynamorphisms work by constructing an intermediate structure with a coalgebra that stores the history of all subresults. One way to interpret this is that the intermediate structure holds a call stack of previous values that can be referenced. While more elaborate functors are supported by the scheme, using a linear structure-such as lists - is a particularly versatile option since we can flatten more complex structures by providing a specific traversal. Using such a traversal ensures that there is sharing between subproblems. The base functor for polymorphic nonempty lists has two constructors: one for when there is a single element, and the other to add elements to the list.

$$
\begin{aligned}
& \text { data List } v x=\text { Some } v \mid \text { Cons } v x \\
& \text { instance Functor (List } v) \text { where } \\
& \text { fmap } f(\text { Some } v)=\text { Some } v \\
& \text { fmap } f(\text { Cons } v x)=\text { Cons } v(f x)
\end{aligned}
$$

To query values from this structure, we develop a number of operations. First, we provide an indexing operator that takes a natural number and returns the corresponding value, working back through the hierarchy the given number of times. The interface is very similar to the indexing operator for standard lists.

$$
\begin{aligned}
& \left(! !_{\infty}\right)::(\text { Show } a, \text { Show } v) \Rightarrow(\text { List } v)_{\infty} a \rightarrow \mathbb{N} \rightarrow a \\
& \left(\text { Cons }_{\infty} a_{-}\right) \quad ! !_{\infty} 0 \quad=a \\
& \left(\text { Cons }_{\infty} a(\text { Cons v as })\right) ! !_{\infty}(n+1)=a s ! !_{\infty} n
\end{aligned}
$$

Of course we could have defined this to be a total function, returning a value of type Maybe $a$ in case the indexing is out of bounds. For our purposes, we use this operator since it reduces clutter in the code that follows.

When more than one value is required at once, and assuming they appear in a contiguous section, it is convenient to make use of the function $t a k e_{\infty} n$, which takes $n$ consecutive values from the hierarchy.

$$
\begin{array}{ll}
\text { take }_{\infty}:: \mathbb{N} \rightarrow(\text { List } v)_{\infty} a \rightarrow[a] & \\
\text { take }_{\infty} 0 & =[] \\
\text { take }_{\infty}(n+1)\left(\text { Cons }_{\infty} a(\text { Some } v)\right) & =[a] \\
\text { take }_{\infty}(n+1)\left(\text { Cons }_{\infty} a(\text { Cons v as })\right) & =a: \text { take } e_{\infty} n \text { as }
\end{array}
$$

Note that it might be tempting to define a related operator, drop $_{\infty}::$ $\mathbb{N} \rightarrow(\text { List } v)_{\infty} a \rightarrow[a]$, which drops a given number of values 
from the hierarchy before returning the values that remain. However, such a definition would be bogus, since we cannot validly use induction over values of type (List $v)_{\infty} v$. The definition of take $e_{\infty}$, however, is perfectly valid, since are using induction over the natural numbers.

With these basic ingredients in place, we are now ready to define some dynamorphisms.

Catalan numbers revisited We now revisit the Catalan numbers, and show how they can be defined in terms of a dynamorphism. The problem we had previously was that there was no means of knowing where in the recursion a call was being made. To store this information, we can define the coalgebra natural as follows:

$$
\begin{aligned}
& \text { natural }:: \mathbb{N} \rightarrow(\text { List } \mathbb{N} \mathbb{N}) \\
& \text { natural } 0=\text { Some } 0 \\
& \text { natural }(n+1)=\text { Cons }(n+1) n
\end{aligned}
$$

To validly apply this in a dynamorphism, we must argue that it is a recursive coalgebra. This amounts to showing that when applied recursively this has the halting property [1]. In this case we observe that in the recursive case, the value $n+1$ is reduced by 1 at each step.

Using this coalgebra, we can form the following definition:

$$
\begin{aligned}
& \text { catalan }_{4}:: \mathbb{N} \rightarrow \mathbb{N} \\
& \text { catalan }_{4}=\text { dyna }_{2} \text { catalan natural where } \\
& \text { catalan }: \text { List } \mathbb{N}\left((\text { List } \mathbb{N})_{\infty} \mathbb{N}\right) \rightarrow \mathbb{N} \\
& \text { catalan (Some 0) }=1 \\
& \text { catalan }(\text { Cons } n \text { table })=\operatorname{sum}(\text { zipWith }(*) \text { xs }(\text { reverse } x s)) \\
& \text { where } x s=\text { take }_{\infty} n \text { table }
\end{aligned}
$$

The key here is that the algebra catalan knows about the current depth of its application, which is held in $n$. Thus, the appropriate number of values can be extracted from the table of previous values, and convoluted just as in previous definitions.

The bitonic travelling-salesman problem We now solve the bitonic travelling-salesman problem using a dynamorphism, and aim for a solution that reflects the algorithm described by bitonic 2 . First we must consider which coalgebra and algebra should be used. The carrier for both of these is already determined by the type of the recursion, and must be $(\mathbb{N}, \mathbb{N})$. What remains to be decided is the base functor for this computation. A crucial part of the solution involves looking up distances between points, and so it is important to keep track of the current point that is being processed during the recursion steps. Therefore, to store these values, we use a base functor of type List $(\mathbb{N}, \mathbb{N})$.

Let us consider the coalgebra that constructs the lookup structure. One of the conditions we can impose is that $i \leqslant j$, since this formed part of our invariant. With this in consideration, it makes sense that only a triangle of values needs to be computed.

$$
\begin{aligned}
& \text { triangle }_{1}::(\mathbb{N}, \mathbb{N}) \rightarrow \text { List }(\mathbb{N}, \mathbb{N})(\mathbb{N}, \mathbb{N}) \\
& \text { triangle }_{1}(0,0)=\text { Some }(0,0) \\
& \text { triangle }_{1}(0,1)=\text { Some }(0,1) \\
& \text { triangle }_{1}(0, j)=\text { Cons }(0, j)(j-1, j-1) \\
& \text { triangle }_{1}(i, j)=\text { Cons }(i, j)(i-1, j)
\end{aligned}
$$

To argue that this coalgebra is recursive, we observe that the reverse lexicographic ordering of the pair $(i, j)$ always decreases, where the relationship with a new pair $\left(i^{\prime}, j^{\prime}\right)$ is given by $(i, j)>\left(i^{\prime}, j^{\prime}\right) \Longleftrightarrow$ $j>j^{\prime} \vee\left(j==j^{\prime} \wedge i>i^{\prime}\right)$. This strategy of building a triangle stores the coordinates in scope as it goes, since this information becomes vital when applying the algebra. Note that here we have included two base cases, both of which follow from the recursive definition of the algorithm.

$$
\begin{aligned}
& \text { bitonic }_{4}::(\mathbb{N}, \mathbb{N}) \rightarrow \text { Double } \\
& \text { bitonic } 4_{4}=\text { dyna }_{2} \text { bitonic triangle } 1 \text { where } \\
& \text { bitonic }:: \text { List }(\mathbb{N}, \mathbb{N})\left((\text { List }(\mathbb{N}, \mathbb{N}))_{\infty} \text { Double }\right) \rightarrow \text { Double } \\
& \text { bitonic }(\text { Some }(0,0))=0 \\
& \text { bitonic }(\text { Some }(0,1))=\overline{x_{0} x_{1}} \\
& \text { bitonic }(\text { Cons }(i, j) \text { table }) \\
& \mid i<j-1=\text { table } ! !_{\infty}(j-1)+\overline{x_{j-1} x_{j}} \\
& \mid i=j-1=\text { minimum } \\
& {\left[\text { table } !_{\infty}(k+j)+\overline{x_{i-k-1} x_{j}} \mid k \leftarrow[0 . . i-1]\right]} \\
& \mid i=j=\text { minimum } \\
& {\left[\text { table } ! !_{\infty} k+\overline{x_{i-k-1} x_{j}} \mid k \leftarrow[0 \ldots i-1]\right]}
\end{aligned}
$$

While this definition has some similarities to the tabular definition in bitonic $_{3}$, the indices are clearly quite different. As with histomorphisms, this is because in this definition we no longer have the ability to make references to previously computed values by using absolute indices: all the indexing into the $t$ structure is relative to the point of call. This is why the 'otherwise' clause from the recursive definition has been split into two different cases.

Chain matrix multiplication The chain matrix multiplication problem also involves computing a triangle of values, since in the definition of chain $_{1} i j$ we have the invariant that $i \leqslant j$. However, the order in which this triangle is built is different to the definition of triangle ${ }_{1}$. For one, we must ensure that $i \geqslant 1$, and furthermore, the access pattern for values in the triangle is somewhat different, since values are instead built from the diagonal to an edge.

$$
\begin{aligned}
& \text { triangle }_{2}::(\mathbb{N}, \mathbb{N}) \rightarrow \operatorname{List}(\mathbb{N}, \mathbb{N})(\mathbb{N}, \mathbb{N}) \\
& \text { triangle }_{2}(1,1)=\text { Some }(1,1) \\
& \text { triangle }_{2}(i, j) \\
& \mid i==j \quad=\text { Cons }(i, j)(1, j-1) \\
& \mid \text { otherwise }^{2}=\text { Cons }(i, j)(i+1, j)
\end{aligned}
$$

Again, we must argue that this coalgebra is recursive, which is only true when $i \leqslant j$. If $i<j$ then $i$ is increased until $i==j$. When this is the case, $i$ is set to 1 and $j$ is decreased by 1 . Thus at any point in the recursion, over the course of at most $j$ steps the value will be reduced to $(1, j-1)$, and eventually this terminates.

The definition of the algebra of chain $_{3}$ requires particular attention to the relative indices: the base case is straight-forward, but when $i<j$ we must calculate the offset carefully.

$$
\begin{aligned}
& \operatorname{chain}_{3}::(\mathbb{N}, \mathbb{N}) \rightarrow \mathbb{N} \\
& \text { chain }_{3}=\text { dyna }_{2} \text { chain triangle } \\
& \text { where } \\
& \text { chain }:: \text { List }(\mathbb{N}, \mathbb{N})\left((\text { List }(\mathbb{N}, \mathbb{N}))_{\infty} \mathbb{N}\right) \rightarrow \mathbb{N} \\
& \text { chain }(\text { Some })=0 \\
& \text { chain }(\text { Cons }(i, j) \text { table }) \\
& \mid i=j=0 \\
& \mid i<j=\text { minimum }\left(\text { zipWith } ( + ) \left[a_{i-1} * a_{k} * a_{j}+\right.\right. \\
& \left.\left.\quad \text { table! } ! !_{\infty} \text { offset } k \mid k \leftarrow[i . j-1]\right]\left(\text { take }_{\infty}(j-i) \text { table }\right)\right) \\
& \text { where } \text { offset } k=((j *(j+1)-k *(k+1)) \text { 'div' } 2)-1
\end{aligned}
$$

To understand this definition, we first note that a cell with index $(i, j)$ is dependent on the cells that are directly 'below' and to the 'left', relative to the ordering imposed in triangle 2 . We consider the cells that immediately precede $(i, j)$ to be below, and those that are found at a particular offset to be to the left. At first glance the definition of offset $k$ may well be mysterious: it arises as a consequence of the triangle numbers $T(n)=\sum_{i=1}^{n} i=n(n+1) / 2$, where we subtract one triangle number from the other: $T(j-1)-$ $T(k)$. This is justified since values to the 'left' are offset by linearly decreasing indices.

\section{Related Work}

Histo- and dynamorphisms The work we have presented builds on the foundations that were set out in the original paper on his- 
tomorphisms [13], where course-of-value iteration was captured as a categorically-inspired recursion scheme. This work was later extended to include dynamorphisms in [9], with the specific goal of extending the reach of histomorphisms to cover dynamic programming algorithms. The authors there also present a number of classic dynamic programming algorithms are given in terms of this framework, and the derivation of efficient dynamorphisms rests on the connection with hylomorphisms. The constructions presented there differ in that they are all within CPO, where initial algebras and final coalgebras coincide.

Recursion schemes from comonads The construction of recursion schemes from comonads was first presented in [14], where the relationship with histomorphisms is explored in detail. That paper also provides an implementation of various recursion schemes in Haskell although it does not make use of type class synonyms, since it predates that work. The correspondence between histomorphisms and recursion schemes from comonads is a direct application of the work in [7], which further explores the relationship between recursion schemes from comonads and the adjoint folds that were developed in [5].

Recursion schemes from recursive coalgebras The notion of obtaining uniqueness properties through recursive coalgebras comes directly from the seminal paper on the topic [2], and a more gentle introduction to recursive coalgebras can be found in [6].

\section{Conclusion}

In this paper we have demonstrated the use of histomorphisms and dynamorphisms through a number of examples, and have shown how these categorically-inspired recursion schemes can be implemented efficiently. The derivation of the efficient versions of histomorphisms relies on their formulation as recursion schemes from comonads and a correspondence between certain bialgebras. Similarly, the derivation of efficient dynamorphisms relies on their formulation as recursion schemes from recursive coalgebras and a correspondence between certain dicoalgebras. These correspondences witness the uniqueness of the constructions through the EilenbergMoore adjunction.

Future work There are a number of avenues for future work. One aspect of dynamorphisms which we have not discussed is the choice of base functor. In this paper, we linearized all structures, and this has required us to pay particular attention to the relative indexing schemes. Another option worth exploring are using a more direct approach such as arrays with a focus. A more structured approach would be to change the base functor to one that both deals with sharing, and that also maintains the structure of the recursion.

Recursive coalgebras are modular in the sense that they can be combined to form even more expressive schemes. For example, it will be convenient to also avail the algebra to the original argument of the function: this arises from considering parametrically recursive coalgebras, which we do not explore here.

\section{References}

[1] J. Adámek, D. Lücke, and S. Milius. Recursive coalgebras of finitary functors. Theoret. Informatics Appl., 41(4):447-462, 2007. doi: 10. 1051/ita:2007028. URL http://dx.doi.org/10.1051/ita: 2007028.

[2] V. Capretta, T. Uustalu, and V. Vene. Recursive coalgebras from comonads. Information and Computation, 204(4):437-468, 2006. doi: 10.1016/j.ic.2005.08.005.

[3] M. M. T. Chakravarty, G. Keller, and S. P. Jones. Associated type synonyms. In Proceedings of the tenth ACM SIGPLAN international conference on Functional programming, ICFP '05, pages 241-253, New York, NY, USA, 2005. ACM. ISBN 1-59593-064-7. doi:
10.1145/1086365.1086397. URL http://doi.acm.org/10. $1145 / 1086365.1086397$.

[4] S. Eilenberg and J. C. Moore. Adjoint functors and triples. Illinois J. Math, 9(3):381-398, 1965.

[5] R. Hinze. Adjoint folds and unfolds-an extended study. Science of Computer Programming, August 2012. doi: 10.1016/j.scico.2012.07. 011. In press.

[6] R. Hinze, D. W. James, and T. Harper. Theory and practice of fusion. In J. Hage and M. Morazán, editors, Proceedings of the 22nd Symposium on the Implementation and Application of Functional Languages (IFL '10), volume 6647 of Lecture Notes in Computer Science, pages 19-37. Springer Berlin / Heidelberg, September 2011. doi: 10.1007/978-3-642-24276-2_2.

[7] R. Hinze, N. Wu, and J. Gibbons. Unifying structured recursion schemes. In International Conference on Functional Programming, March 2013. URL http://www.cs.ox.ac.uk/ people/jeremy.gibbons/publications/urs.pdf. Accepted for publication.

[8] P. J. Huber. Homotopy theory in general categories. Mathematische Annalen, 144:361-385, 1961. URL http://dx.doi.org/10. $1007 / \mathrm{BF} 01396534$. 10.1007/BF01396534.

[9] J. Kabanov and V. Vene. Recursion schemes for dynamic programming. In Mathematics of Program Construction, 8th International Conference, MPC 2006, pages 235-252. Springer, 2006.

[10] H. Kleisli. Every standard construction is induced by a pair of adjoint functors. Proceedings of the American Mathematical Society, 16(3): 544-546, June 1965. URL http://www. jstor.org/stable/ 2034693.

[11] J. Lambek. A fixpoint theorem for complete categories. Math. Zeitschr., 103:151-161, 1968.

[12] S. Peyton Jones. Haskell 98 Language and Libraries. Cambridge University Press, 2003.

[13] T. Uustalu and V. Vene. Primitive (co)recursion and course-of-value (co)iteration, categorically. Informatica, Lith. Acad. Sci., 10(1):5-26, 1999.

[14] T. Uustalu, V. Vene, and A. Pardo. Recursion schemes from comonads. Nordic J. of Computing, 8:366-390, September 2001. 